\title{
Cooperative Diversity in Wireless Relay Networks with Multiple-Antenna Nodes
}

\author{
Yindi Jing \\ Department of Electrical Engineering \\ California Institute of Technology \\ Pasadena, CA 91125, U.S.A. \\ Email: yindi@systems.caltech.edu
}

\author{
Babak Hassibi \\ Department of Electrical Engineering \\ California Institute of Technology \\ Pasadena, CA 91125, U.S.A. \\ Email: hassibi@systems.caltech.edu
}

\begin{abstract}
In [1], the idea of distributed space-time coding was proposed to achieve a degree of cooperative diversity in a wireless relay network. In particular, for a relay network with a single-antenna transmitter and receiver and $R$ single-antenna relays, it was shown that the pairwise error probability (PEP) decays as $\left(\frac{\log P}{P}\right)^{R}$, where $P$ is the total transmit power. In this paper, we extend the results to wireless relay networks where the transmitter, receiver, and/or relays may have multiple antennas. Assuming that the transmitter has $M$ antennas, the receiver has $N$ antennas, the sum of all the antennas at the relay nodes is $R$, and the coherence interval is long enough, we show that the PEP behaves as $\left(\frac{1}{P}\right)^{\min \{M, N\} R}$, if $M \neq N$, and $\left(\frac{\log ^{1 / M} P}{P}\right)^{M R}$, if $M=N$. Therefore, for the case of $M \neq N$, distributed spacetime coding has the same PEP performance as a multiple-antenna system with $\min \{M, N\} R$ transmit and a single receive antenna. For the case of $M=N$, the penalty on the PEP compared to a multiple-antenna system is a $\log ^{1 / M} P$ factor, which is negligible at high SNR. We also show that for a fixed total transmit power across the entire network, the optimal power allocation is for the transmitter to expend half the power and for the relays to share the other half with the power used by each relay being proportional to the number of antennas it has.
\end{abstract}

\section{INTRODUCTION}

It is known that multiple antennas can greatly increase the capacity and reliability of a wireless communication link in a fading environment using space-time codes [2], [3]. Recently, with the increasing interests in ad hoc networks, researchers have been looking for methods to exploit spatial diversity using the antennas of different users in the network [4]-[9], [1]. In most of these works, decoding is needed at the relay nodes, which puts a heavy constraint on the transmission rate especially when the number of relay nodes is large. It also causes extra time delay and power consumption because of decoding complexity.

In [1], the idea of space-time coding devised for multipleantenna systems is applied to the problem of communication over a wireless relay network with single-antenna nodes. ${ }^{1}$ The authors consider wireless relay networks with fading and use a cooperative strategy called distributed space-time coding by applying a linear dispersion space-time code [10] among the relays. It is proved that without any channel knowledge at the

${ }^{1}$ Although having the same name, the distributed space-time coding idea in [1] is different from that in [6]. Similar ideas for networks with two relay nodes appeared in [7], [9]. relay nodes, for wireless relay networks with $R$ single-antenna nodes, distributed space-time coding achieves the diversity of a multiple-antenna system with $R$ transmit antennas and one receive antenna asymptotically. That is, the relay nodes work as transmit antennas of the transmit node although they cannot fully cooperate and do not have full knowledge of the transmit signal. Compared with the other widely used cooperative strategy, decode-and-forward, since no decoding is needed at the relay nodes, distributed space-time coding saves both time and energy and more importantly, there is no rate constraint on the transmission.

In this paper, we extend the idea of distributed space-time coding to wireless relay networks whose nodes may have multiple antennas. We use the same two-phase transmission method in [1] where in one phase the transmitter sends signals to the relay nodes and in the other the relays encode their received signals into a linear dispersion space-time code and transmit to the receiver. For a wireless relay network with $M$ antennas at the transmit node, $N$ antennas at the receive node, and a total of $R$ antennas at all the relay nodes, our work show that when the coherence interval is long enough, using similar distributed space-time coding as in [1], the PEP of the network behaves as $\left(\frac{1}{P}\right)^{\min \{M, N\} R}$ if $M \neq N$ and $\left(\frac{\log ^{1 / M} P}{P}\right)^{M R}$ if $M=N$, where $P$ is the total transmit power used in the whole network. With the two-phase strategy, it is easy to see that that no matter what cooperative strategy is used at the relay nodes, the diversity of the first phase of transmission, i.e. from the transmitter to the relays, cannot be larger than $M R$, the diversity of a multiple-antenna system with $M$ transmit antennas and $R$ receive antennas; similarly, the diversity of the second phase, i.e. transmission from the relay nodes to the receiver, cannot be larger than $N R$. Therefore, when $M \neq N$, distributed space-time coding is optimal according to diversity. For the case of $M=N$, the penalty on the PEP is just a $\log ^{1 / M} P$ factor. Therefore, with distributed space-time coding, wireless relay networks achieve the same diversity of multiple-antenna systems, asymptotically.

\section{Wireless Relay Network Model}

We first introduce some notations used in the paper. For a complex matrix $A, A^{t}, A^{*}$, and $A^{-1}$ denote the transpose, the 


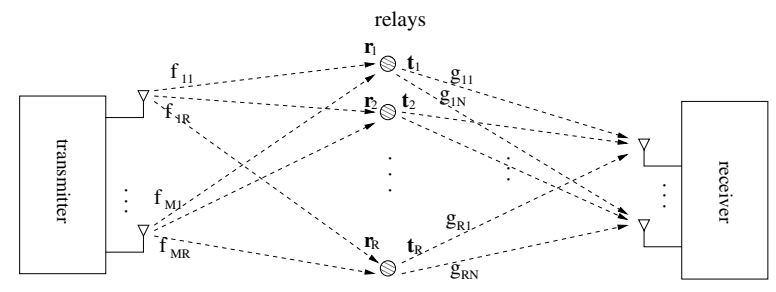

Fig. 1. Wireless relay network with multiple-antenna nodes

conjugate transpose, and the inverse of $A$, respectively. $\operatorname{det} A$ and $\operatorname{tr} A$ indicate the determinant and trace of $A$, respectively. $I_{n}$ denotes the $n \times n$ identity matrix and $\operatorname{diag}\left\{a_{1}, \cdots, a_{n}\right\}$ is the $n \times n$ diagonal matrix with $a_{1}, \cdots, a_{n}$ being its diagonal entries. $\log$ indicates the natural logarithm. $\mathrm{P}$ and $\mathrm{E}$ indicate the probability and the expected value.

Consider a wireless network with $R+2$ nodes which are placed randomly and independently according to some distribution. There is one transmit node and one receive node. All the other $R$ nodes work as relays. This is a practical model for many sensor networks. The transmitter has $M$ transmit antennas, the receiver has $N$ receive antennas, and the $i$ th relay node has $R_{i}$ antennas, which can be used for both transmission and reception. Since the transmit and receive signals at different antennas of the same relay can be processed and designed independently, this network can be transformed to a wireless relay network with $\sum_{i=1}^{R} R_{i}$ single-antenna relay nodes. Therefore, without loss of generality, in our paper, we always assume that every relay node has a single antenna.

Therefore, the network can be depicted by Figure 1. Denote the channels from the $M$ antennas of the transmitter to the $i$ th relay as $f_{1 i}, \cdots, f_{M i}$, and the channels from the $i$-th relay to the $N$ antennas at the receiver as $g_{i 1}, \cdots, g_{i N}$. Here, we only consider the fading effect by assuming that $f_{m i}$ and $g_{i n}$ are independent complex Gaussian with zero-mean and unitvariance, $\mathcal{C N}(0,1)$. This is a common assumption for urban areas when there is no line-of-sight or when the distances between relay nodes and the transmitter/receiver are about the same. We make the practical assumption that the channels $f_{m i}$ and $g_{i n}$ are not known at the $i$-th relays. What every relay knows is just the statistical distribution of its local connections. However, we do assume that the receiver knows $f_{m i}$ and $g_{i n}$. Its knowledge of the channels can be obtained by sending training signals from the relays and the transmitter.

Assume that the transmitter wants to send the signal $\underline{\mathbf{s}}=$ $\left[\mathbf{s}_{1}, \cdots, \mathbf{s}_{M}\right]$ to the receiver. Here, $\mathbf{s}_{m}$, is a $T$-dimensional vector and is the signal sent by the $m$-th transmit antenna. $T$ is the coherence interval, that is, the time duration among which the channels $f_{m i}$ and $g_{i n}$ keep constant. ${ }^{2}$ Therefore $\underline{\mathbf{s}}$ is a $T \times M$ matrix. As in multiple-antenna systems [2], we always assume that $T \geq M$. $\underline{\mathbf{s}}$ can be either the uncoded information bits or an element from any coded signal set. $\underline{\mathbf{s}}$ is normalized

${ }^{2}$ From the protocol discussed in the following, we can see that since we only need $f_{m i}$ to keep constant for the first step of the transmission and $g_{i n}$ to keep constant for the second step, it is good enough to choose $T$ as the minimum of the coherence intervals of $f_{m i}$ and $g_{i n}$. as

$$
\operatorname{Etr} \underline{\mathbf{s}}^{*} \underline{\mathbf{s}}=M .
$$

To accomplish this transmission goal, the same two-step strategy in [1] is used here. In step one, which is from time 1 to $T$, the transmitter sends signals $\sqrt{P_{1} T / M} \underline{\mathbf{s}}$ with $\sqrt{P_{1} T / M} \mathbf{s}_{m}$ the signal sent by the $m$-th antenna. From (1), it is easy to see that the average total power used at the transmitter is $P_{1} T$. We denote the received signal vector and the noise vector from time 1 to $T$ at the $i$-th relay node as $\mathbf{r}_{i}$ and $\mathbf{v}_{i}$. In step two, which is from time $T+1$ to $2 T$, the $i$-th relay transmits vector $\mathbf{t}_{i}$ to the receiver based on its received signals. We denote the received signal and noise at the $n$-th receive antenna of the receiver from time $T+1$ to $2 T$ by $\mathbf{x}_{n}$ and $\mathbf{w}_{n}$. It is easy to see that $\mathbf{v}_{i}, \mathbf{r}_{i}, \mathbf{t}_{i}, \mathbf{w}_{n}, \mathbf{x}_{n}$ are all $T$-dimensional column vectors. Assume that the noises are complex Gaussian with zero-mean and unit-variance, that is, entries of $\mathbf{v}_{i}, \mathbf{w}_{n}$ are $\mathcal{C N}(0,1)$.

While $f_{m i}$ and $g_{i n}$ keep constant for $T$ transmissions, clearly

$$
\begin{aligned}
& \qquad \mathbf{r}_{i}=\sqrt{P_{1} T / M} \underline{\mathbf{s}}_{i}+\mathbf{v}_{i} \text { and } \mathbf{x}_{n}=\sum_{i=1}^{R} g_{i n} \mathbf{t}_{i}+\mathbf{w}_{n}, \\
& \text { where we have defined } \mathbf{f}_{i}=\left[\begin{array}{c}
f_{1 i} \\
\vdots \\
f_{M i}
\end{array}\right] .
\end{aligned}
$$

\section{Distributed Space-Time Coding}

We want to have the relay nodes in the network cooperate in a way such that the antennas of the relays work as transmit antennas of the transmitter to obtain diversity. There are two main differences between the wireless network in Figure 1 and a multiple-antenna system. The first one is that in the latter, antennas of the transmitter can cooperate fully while in the former, the relays do not communicate with each other and can only cooperate in a distributed fashion. The other difference is that in the wireless network, every relay node only has a noisy version of the transmit signal.

The main issue is what the relays should do. One of the most widely used strategies is called decode-and-forward, in which the $i$-th relay first obtains an estimate of the transmit signal, $\underline{\hat{\mathbf{s}}}_{i}$, by fully decoding its received signal $\mathbf{r}_{i}$, and then encodes the information again and transmits the newly encoded signal. If the transmission rate is sufficiently low so that all the $R$ relays decode correctly, the second step of transmission is equivalent to the transmission of a multiple-antenna systems with $R$ transmit antennas and $N$ receive antennas, whose maximum achievable diversity is $N R$. However, if some relays decode incorrectly, they will forward incorrect information to the receiver, which will harm the decoding at the receiver greatly. Therefore, to use decode-and-forward, the transmission rate should be low enough to allow all the $R$ relays to decode correctly. Therefore, decode-and-forward requires a substantial reduction of the rate especially for large $R$, and we will therefore not consider it here. There are other disadvantages 
of decode-and-forward: because of the decoding complexity, it causes both extra time delay and energy consumption.

In this paper, we will use the cooperative strategy called distributed space-time coding proposed in [1], which does not require the relay nodes to decode. Design the transmit signal at relay $i$ as

$$
\mathbf{t}_{i}=\sqrt{\frac{P_{2}}{P_{1}+1}} A_{i} \mathbf{r}_{i}
$$

a linear function of its received signal. $A_{i}$ is a $T \times T$ unitary matrix. Similar to [1], while within the framework of linear dispersion codes, $A_{i}$ can be arbitrary, to have a protocol that is equitable among different users and among different time instants, we set $A_{i}$ to be unitary. This also simplifies the analysis on the PEP considerably.

Since $f_{m i}, v_{i, j}$ are $\mathcal{C N}(0,1)$, and $f_{m i}, \underline{\mathbf{s}}, v_{i, j}$ are independent, with the normalization in (1), it is easy to see that the average transmit power at every relay is $P_{2} T$, which explains our normalization in (3). $P_{2}$ is the average transmit power for one transmission at every relay.

Let us now focus on the received signal. Clearly from (2) we can obtain the received signal at the $n$-th receive antenna:

$$
\begin{aligned}
& \mathbf{x}_{n}=\sqrt{\frac{P_{1} P_{2} T}{\left(P_{1}+1\right) M}}\left[\begin{array}{lll}
A_{1} \underline{\mathbf{s}} & \cdots & A_{R} \underline{\mathbf{s}}
\end{array}\right]\left[\begin{array}{c}
\mathbf{f}_{1} g_{1 n} \\
\vdots \\
\mathbf{f}_{R} g_{R n}
\end{array}\right] \\
& +\sqrt{\frac{P_{2}}{P_{1}+1}} \sum_{i=1}^{R} g_{i n} A_{i} \mathbf{v}_{i}+\mathbf{w} .
\end{aligned}
$$

By defining

$$
\begin{aligned}
X=\left[\begin{array}{lll}
\mathbf{x}_{1} & \cdots & \mathbf{x}_{N}
\end{array}\right], & \mathbf{g}_{i}=\left[\begin{array}{lll}
g_{i 1} & \cdots & g_{i N}
\end{array}\right], \\
S=\left[\begin{array}{lll}
A_{1} \underline{\mathbf{s}} & \cdots & A_{R} \underline{\mathbf{s}}
\end{array}\right], & H=\left[\begin{array}{c}
\mathbf{f}_{1} \mathbf{g}_{1} \\
\vdots \\
\mathbf{f}_{R} \mathbf{g}_{R}
\end{array}\right],
\end{aligned}
$$

and

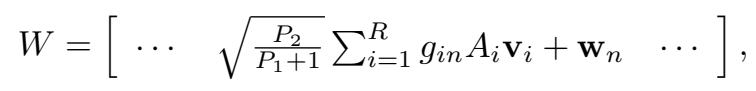

the system equation can be written as

$$
X=\sqrt{\frac{P_{1} P_{2} T}{\left(P_{1}+1\right) M}} S H+W .
$$

Let's look at the dimensions of each matrix. $X$, which is the received signal matrix, is $T \times N . S$ is $T \times M R$, since $A_{i}$ are $T \times T, \underline{\mathbf{s}}$ is $T \times M$, and there are $R$ of them. $\mathbf{f}_{i}$ is $M \times 1$ and $\mathrm{g}_{i}$ is $1 \times N$. Therefore, the equivalent channel matrix $H$ is $R M \times N$. W, which is the equivalent noise matrix, is $T \times N$. Similar to [1], $S$ works like the space-time code in a multiple-antenna system. It is called the distributed space-time code since it has been generated in a distributed way by the relays without having access to the transmit signal. The power constraint on the space-time code $S$ is:

$$
\mathrm{E} \operatorname{tr} S^{*} S=\mathrm{E} \sum_{i=1}^{R} \operatorname{tr} \underline{\mathbf{s}}^{*} A_{i}^{*} A_{i} \underline{\mathbf{s}}=R \operatorname{E\operatorname {tr}} \underline{\mathbf{s}}^{*} \underline{\mathbf{s}}=M R .
$$

\section{Pairwise Error Probability}

To analyze the PEP, we have to obtain the maximumlikelihood (ML) decoding, which needs $\mathrm{P}\left(X \mid \underline{\mathbf{s}}_{k}\right)$, where $\underline{\mathbf{s}}_{k} \in$ $\mathcal{S}$ the set of all possible transmit signals. The following theorem is straightforward.

Theorem 1: Suppose $\underline{\mathbf{s}}_{k}$ is transmitted and define

$$
S_{k}=\left[\begin{array}{llll}
A_{1} \underline{\mathbf{s}}_{k} & A_{2} \underline{\mathbf{s}}_{k} & \cdots & A_{R} \underline{\mathbf{s}}_{k}
\end{array}\right] .
$$

Then subject to $\underline{\mathbf{s}}_{k}$ being transmitted, the rows of $X$ are independently Gaussian distributed with the same variance $I_{N}+\frac{P_{2}}{P_{1}+1} G G^{*}$, where

$$
G=\left[\begin{array}{ccc}
g_{11} & \cdots & g_{R 1} \\
\vdots & \ddots & \vdots \\
g_{1 N} & \cdots & g_{R N}
\end{array}\right]
$$

is a $N \times R$ matrix. The $t$-th row of $X$ has mean $\sqrt{\frac{P_{1} P_{2} T}{M\left(P_{1}+1\right)}}\left[S_{k}\right]_{t} H$, where $\left[S_{k}\right]_{t}$ is the $t$-th row of $S_{k}$.

Therefore, we can easily write down the probability density function (pdf) of $X \mid \underline{\mathbf{s}}_{k}$, which is the product of the pdf of $\mathbf{x}_{n} \mid \underline{\mathbf{s}}_{k}$ for all $n=1, \cdots, N$. We should emphasis that from this theorem, we can see that, unlike multiple-antenna systems, the received signals at different antennas, that is, the columns of $X$, are not independent. However, the received signals at different times continue to be independent. Also, unlike the case of relay network with single antenna at the receiver, where the conditional received signals are white Gaussian, here, although they are still Gaussian, they are no longer white since $G G^{*}$ is not diagonal in general. This makes the PEP analysis in this paper much more difficult than that in [1].

We have proved the following theorem.

Theorem 2 (ML decoding and Chernoff bound on PEP): Define $R_{w}=I_{N}+\frac{P_{2}}{P_{1}+1} G G^{*}$. The ML decoding of the network is

$\arg \min _{\underline{\mathbf{s}}_{k}} \operatorname{tr}\left(X-\sqrt{\frac{P_{1} P_{2} T}{M\left(P_{1}+1\right)}} S_{k} H\right) R_{w}^{-1}\left(X-\sqrt{\frac{P_{1} P_{2} T}{M\left(P_{1}+1\right)}} S_{k} H\right)^{*}$.

With this ML decoding, the PEP of mistaking $\underline{\mathbf{s}}_{k}$ by $\underline{\mathbf{s}}_{l}$, averaged over the channel distributions, has the following upper bound:

$$
P E P \leq \underset{f_{m i}, g_{i n}}{\mathrm{E}} e^{-\frac{P_{1} P_{2} T}{4 M\left(1+P_{1}\right)} \operatorname{tr}\left(S_{k}-S_{l}\right)^{*}\left(S_{k}-S_{l}\right) H R_{w}^{-1} H^{*}}
$$

\section{Power Allocation}

The main purpose of this work is to analyze how the PEP decays with the total power consumed in the whole network. We set the total power used in the whole network as $P$. Therefore, $P=P_{1}+R P_{2}$. In this section, we discuss the optimum power allocation between the transmitter and the relay nodes such that the PEP is minimized. Because of the expectations over $f_{m i}$ and $g_{i n}$ and also the dependency of $R_{w}$ on $g_{i n}$, the exact minimization of (6) is very difficult. Therefore, similar to the argument in [1], we recourse to an asymptotic argument at $R \rightarrow \infty$. 
Notice that the $(m, n)$-th entry of $\frac{1}{R} G G^{*}$ is $\frac{1}{R} \sum_{i=1}^{R} g_{i m} \bar{g}_{i n}$. When $R \rightarrow \infty$, according to the law of large numbers, the off-diagonal entries of $\frac{1}{R} G G^{*}$ goes to zero while the diagonal entries approach to 1 with probability 1 since $\sum_{i=1}^{R}|g|_{i n}^{2}$ has a gamma distribution with both mean and variance $R$. Therefore, it is reasonable to make the assumption $G G^{*} \approx R I_{N}$ for large $R$, which is the same as $R_{w} \approx\left(1+\frac{P_{2} R}{P_{1}+1}\right) I_{N}$.

Therefore, from (6),

$$
P E P \lesssim \underset{f_{m i}, g_{i n}}{\mathrm{E}} e^{-\frac{P_{1} P_{2} T}{4 M\left(1+P_{1}+R P_{2}\right)} \operatorname{tr}\left(S_{k}-S_{l}\right)^{*}\left(S_{k}-S_{l}\right) H H^{*}}
$$

Since $S_{k}, S_{l}$, and $H$ are independent of $P_{1}$ and $P_{2}$, minimizing the PEP is equivalent to maximizing $\frac{P_{1} P_{2} T}{4 M\left(1+P_{1}+R P_{2}\right)}$. This is exactly the same power allocation problem that appeared in [1]. Therefore, with the same argument, we can conclude that the optimum solution is to set

$$
P_{1}=\frac{P}{2} \quad \text { and } \quad P_{2}=\frac{P}{2 R} .
$$

Thus, the optimum power allocation is such that the transmitter uses half the total power and the relays share the other half fairly. When the number of relays is large, which is the case for many sensor networks, every relay spends only a very small amount of power to help the transmitter. ${ }^{3}$

Note that as discussed in Section II, for wireless relay networks with $R_{i}$ antennas at the $i$-th relay, the $R_{i}$ antennas are treated as $R_{i}$ different single-antenna relays. Therefore, it is easy to see that for this multiple-antenna-relay-node case, the optimum power allocation is such that the transmitter uses half the total power, but every relay node uses power that is proportional to its number of antennas. That is $P_{1}=\frac{P}{2}$ and the power used at the $i$-th relay is $\frac{R_{i} P}{2 R}$.

With this power allocation, at high $P$, we have

$$
P E P \leq \underset{f_{m i}, g_{i n}}{\mathrm{E}} e^{-\frac{P T}{16 M R} \operatorname{tr}\left(S_{k}-S_{l}\right)^{*}\left(S_{k}-S_{l}\right) H H^{*}} .
$$

\section{Diversity AnAlysis For $R \rightarrow \infty$}

As mentioned earlier, to obtain the diversity, we have to compute the expectations over $f_{m i}$ and $g_{i n}$ in (8). Here we give a simple asymptotic derivation for the case of large number of relay nodes, that is $R \rightarrow \infty$. For the general case, refer to [13]. As discussed in the previous section, when $R$ is large, we can make the approximation

$$
R_{w} \approx\left(1+\frac{P_{2} R}{P_{1}+1}\right) I_{N}
$$

and obtain (8). Denote the $n$-th column of $H$ as $\mathbf{h}_{n}$. From (4), we can see that $\mathbf{h}_{n}=\mathcal{G}_{n} \mathbf{f}$, where we have defined $\mathcal{G}_{n}=$ $\operatorname{diag}\left\{g_{1 n} I_{M}, \cdots, g_{R n} I_{M}\right\}$ and $\mathbf{f}=\left[\begin{array}{c}\mathbf{f}_{1} \\ \vdots \\ \mathbf{f}_{R}\end{array}\right]$. Therefore, (8) can be equivalently written as

$$
P E P \lesssim \underset{f_{m i}, g_{i n}}{\mathrm{E}} e^{-\frac{P T}{16 M R} \mathbf{f}^{*}\left[\sum_{n=1}^{N} \mathcal{G}_{n}^{*}\left(S_{k}-S_{l}\right)^{*}\left(S_{k}-S_{l}\right) \mathcal{G}_{n}\right] \mathbf{f}} .
$$

${ }^{3}$ This power allocation is obtained for the network model where the pathloss of the channels is not taken into account.
Since $\mathbf{f}$ is white Gaussian with mean zero and variance $I_{R M}$, we can calculate the expectation over $\mathbf{f}$ to obtain:

$P E P \lesssim \underset{g_{i n}}{\mathrm{E}} \operatorname{det}^{-1}\left[I_{R M}+\frac{P T}{16 M R} \sum_{n=1}^{N} \mathcal{G}_{n}^{*}\left(S_{k}-S_{l}\right)^{*}\left(S_{k}-S_{l}\right) \mathcal{G}_{n}\right]$.

Similar to the multiple-antenna case [3], [11] and the case of wireless relay networks with single-antenna nodes [1], the "full diversity" condition can be obtained. It is easy to see that if $S_{k}-S_{l}$ drops rank, the exponent of $P$ in the right side of (10) increases. That is, the diversity decreases. Therefore, the Chernoff bound is minimized when $S_{k}-S_{l}$ is full-rank, or equivalently, $\operatorname{det}\left(S_{k}-S_{l}\right) *\left(S_{k}-S_{l}\right) \neq 0$ for all $S_{k} \neq S_{l} \in \mathcal{S}$. Since the distributed space-time code $S_{k}$ and $S_{l}$ are $T \times M R$, there is no point in having $M R$ larger than the coherence interval $T$. Thus, in the following, we will always assume $T \geq M R$.

Assume that the code is fully-diverse. It is easy to see from (10) that, roughly speaking, the larger the positive matrix $\left(S_{k}-S_{l}\right)^{*}\left(S_{k}-S_{l}\right)$, the smaller the upper bound. This improvement in the PEP according to the optimization of the distributed space-time code is called coding gain. Here, to highlight the diversity result, we shall neglect the affect of the design of the distributed space-time code and analyze the diversity gain obtained from the independent transmission routes from antennas of the transmitter to antennas of the receiver via the relay nodes. To do so, we shall look at the behavior of the PEP at high SNR. Such an analysis is consistent with the diversity definition of multiple-antenna systems in [3], [11]. ${ }^{4}$

Denote the minimum singular value of $\left(S_{k}-S_{l}\right) *\left(S_{k}-\right.$ $\left.S_{l}\right)$ as $\sigma_{\min }^{2}$. From the full diversity of the code, $\sigma_{\min }^{2}>0$. Therefore, the right side of formula (10) can be further upper bounded as the following:

$$
\begin{aligned}
P E P & \lesssim \underset{g_{\text {in }}}{\mathrm{E}} \operatorname{det}^{-1}\left[I_{T M}+\frac{P T \sigma_{\min }^{2}}{16 M R} \sum_{n=1}^{N} \mathcal{G}_{n}^{*} \mathcal{G}_{n}\right] \\
& =\underset{g_{\text {in }}}{\mathrm{E}} \prod_{i=1}^{R}\left(1+\frac{P T \sigma_{\min }^{2}}{16 M R} \sum_{n=1}^{N}\left|g_{i n}\right|^{2}\right)^{-M} .
\end{aligned}
$$

Define $g_{i}=\sum_{n=1}^{N}\left|g_{i n}\right|^{2}$. Since all $g_{i n}$ are i.i.d. $\mathcal{C N}(0,1)$, $g_{i}$ are i.i.d. gamma distributed with mean $N$, whose pdf is $p\left(g_{i}\right)=\frac{1}{(N-1) !} g_{i}^{N-1} e^{-g_{i}}$. Therefore,

$$
P E P \lesssim \frac{1}{(N-1) !^{R}}\left[\int_{0}^{\infty} \frac{x^{N-1} e^{-x}}{\left(1+\frac{P T \sigma_{\min }^{2}}{16 M R}\right)^{M}} d x\right]^{R},
$$

which can be calculated directly to obtain Theorem 3. Of course, our derivation here is not rigorous since it is predicated

\footnotetext{
${ }^{4}$ However, it is different from the explicit diversity definition, $-\lim _{P \rightarrow \infty} \frac{\log P E P}{\log P}$, given in [14]. It is more precise in the sense that it sometimes describes the PEP behavior in more detail. For example, if $P E P \sim P^{-[c+f(P)]}$, the same diversity $c$ will be obtained for any function $f$ satisfying $\lim _{P \rightarrow \infty} f(P)=0$.
} 
on the approximation in (9). However, in [13] we show that the result is true even if we perform a rigorous analysis.

Theorem 3 (Diversity gain for large $R$ ): Assume that $R \gg N$ and $T \geq M R$. At high total transmit power $P$, by looking at only the highest order term of $P$, the PEP of mistaking $\underline{\mathbf{s}}_{k}$ with $\underline{\mathbf{s}}_{l}$, averaged over the channel distributions, has the following upper bound:

$P E P \lesssim \begin{cases}{\left[\frac{2^{N-1}}{(M-N)(N-1) !}\right]^{R}\left(\frac{16 M R}{T \sigma_{\min }^{2}}\right)^{N R}\left(\frac{1}{P}\right)^{N R} \text { if } M>N} \\ \frac{1}{(N-1) ! R}\left(\frac{16 M R}{T \sigma_{\min }^{2}}\right)^{M R}\left(\frac{\log ^{1 / M} P}{P}\right)^{M R} & \text { if } M=N \\ {\left[\frac{(N-M-1) !}{(N-1) !}\right]^{R}\left(\frac{16 M R}{T \sigma_{\min }^{2}}\right)^{M R}\left(\frac{1}{P}\right)^{M R}} & \text { if } M<N\end{cases}$

The diversity of the wireless relay network is therefore

$$
d=\left\{\begin{array}{ll}
\min \{M, N\} R & \text { if } M \neq N \\
M R\left(1-\frac{1}{M} \frac{\log \log P}{\log P}\right) & \text { if } M=N
\end{array} .\right.
$$

Of course, with the diversity definition in [14], all cases exhibit the full diversity of $\min \{M, N\} R$.

With the two-step protocol, it is easy to see that regardless of the cooperative strategy used at the relay nodes, the error probability is determined by the worse of the two transmission stages: the transmission from the transmitter to the relays and the transmission from the relays to the receiver. It is also easy to see that the PEP of the first stage cannot be better than the PEP of a multiple-antenna system with $M$ transmit antennas and $R$ receive antennas, which has diversity $M R$, while the PEP of the second stage can have diversity no larger than $N R$. Therefore, when $M \neq N$, according to diversity based on the PEP, distributed space-time coding is optimal. For the case of $M=N$, the penalty on the PEP is just a $\log ^{1 / M} P$ factor, which is negligible when $P$ is high. Therefore, distributed space-time coding is better than decode-and-forward since it achieves the optimum diversity gain without the rate constraint needed for decode-and-forward.

\section{CONCLUSION AND Future Work}

In this paper, we extend the idea of distributed space-time coding to communications in wireless relay networks with multiple-antenna nodes. The ML decoding and PEP at the receiver are analyzed. For a wireless relay network with $M$ antennas at the transmitter, $N$ antennas at the receiver, a total of $R$ antennas at all the relay nodes, and a coherence interval no less than $M R$, our main result is that the diversity behaves as $\min \{M, N\} R$, if $M \neq N$, and $M R\left(1-\frac{1}{M} \frac{\log \log P}{\log P}\right)$, if $M=N$, where $P$ is the total power used in the whole network. Although the result is based on an assumption that is valid for large $R$, a more rigorous derivation in [13] gives the same diversity result for general $R$. This result shows the optimality of distributed space-time coding according to the diversity gain.

We also show that, for a fixed total transmit power across the entire network, the optimal power allocation is for the transmitter to expand half the power and for the relays share the other half such that the power used by each relay is proportional to the number of antennas it has.

There are several directions for future work that can be envisioned. Firstly, in addition to the diversity gain, coding gain or the design of the space-time code

$$
S=\left[\begin{array}{lll}
A_{1} \underline{\mathbf{s}} & \cdots & A_{R} \underline{\mathbf{s}}
\end{array}\right]
$$

is also very important to the performance of the wireless relay network. This is under current investigation.

Another important problem is the non-coherent case. In this work, we assume that the receiver knows all the channel information, which needs training from both the transmitter and the relay nodes. For networks with high mobility and/or a large number of relay nodes, this is not a practical assumption. Therefore, it should be interesting to see whether differential space-time coding techniques can be generalized to the distributed wireless relay network.

\section{ACKNOWLEDGMENT}

This work is supported in part by the National Science Foundation under grant numbers CCR-0133818 and CCR0326554, by the David and Lucille Packard Foundation, and by Caltech's Lee Center for Advanced Networking.

\section{REFERENCES}

[1] Y. Jing and B. Hassibi, "Distributed space-time coding in wireless relay networks" Submitted to IEEE Transactions on Communications, 2004.

[2] T. L. Marzetta and B. M. Hochwald, "Capacity of a mobile multipleantenna communication link in Rayleigh flat fading," IEEE Trans. Info. Theory, vol. 45, pp. 139-157, Jan. 1999.

[3] V. Tarokh, N. Seshadri, and A. R. Calderbank, "Space-time codes for high data rate wireless communication: Performance criterion and code construction," IEEE Trans. Info. Theory, vol. 44, pp. 744-765, 1998.

[4] A. Sendonaris, E. Erkip, and B. Aazhang, "User cooperation diversitypart I: System description," IEEE Transactions on Communications, vol. 51, pp. 1927-1938, Nov. 2003.

[5] A. Sendonaris, E. Erkip, and B. Aazhang, "User cooperation diversitypart II: Implementation aspects and performance analysis," IEEE Transactions on Communications, vol. 51, pp. 1939-1948, Nov. 2003.

[6] J. N. Laneman and G. W. Wornell, "Distributed space-time-coded protocols for exploiting cooperative diversity in wireless network," IEEE. Trans. on Info. Theory, vol. 49, pp. 2415-2425, Oct. 2003.

[7] R. U. Nabar, H. Bolcskei, and F. W. Kneubuhler, "Fading relay channels: Performance limits and space-time signal design," IEEE Journal on Selected Areas in Communications, vol. 22, pp. 1099-1109, Aug. 2004.

[8] Y. Hua, Y. Mei, and Y. Chang, "Wireless antennas-making wireless communications perform like wireline communications," in IEEE AP-S Topical Conference on Wireless Communication Technology, Oct. 2003.

[9] Y. Chang and Y. Hua, "Application of space-time linear block codes to parallel wireless relays in mobile ad hoc networks," in the Thirty-Sixth Asilomar Conference on Signals, Systems and Computers, Nov. 2003.

[10] B. Hassibi and B. Hochwald, "High-rate codes that are linear in space and time," IEEE Trans. Info. Theory, vol. 48, pp. 1804-1824, July 2002.

[11] B. M. Hochwald and T. L. Marzetta, "Unitary space-time modulation for multiple-antenna communication in Rayleigh flat-fading," IEEE Trans. Info. Theory, vol. 46, pp. 543-564, Mar. 2000.

[12] I. S. Gradshteyn and I. M. Ryzhik, Table of Integrals, Series and Products. Academic Press, 6nd ed., 2000.

[13] Y. Jing and B. Hassibi, "Cooperative diversity in wireless relay networks with multiple-antenna nodes," In preparation.

[14] L. Zheng and D. Tse, "Diversity and multiplexing: a fundamental tradeoff in multiple antenna channels," IEEE Transactions on Information Theory, vol. 49, pp. 1073-1096, May 2003. 